MPIKG Public Access

Author Manuscript

Published in final edited form as:

Pieber, B., Shalom, M., Antonietti, M., Seeberger, P. H., \& Gilmore, K. (2018). Continuous Heterogeneous Photocatalysis in Serial Micro-Batch Reactors. Angewandte Chemie International Edition, 130(31), 10127-10131. doi:10.1002/ange.201712568.

\title{
Continuous Heterogeneous Photocatalysis in Serial Micro-Batch Reactors
}

Bartholomäus Pieber, Menny Shalom, Markus Antonietti, Peter H. Seeberger, Kerry Gilmore

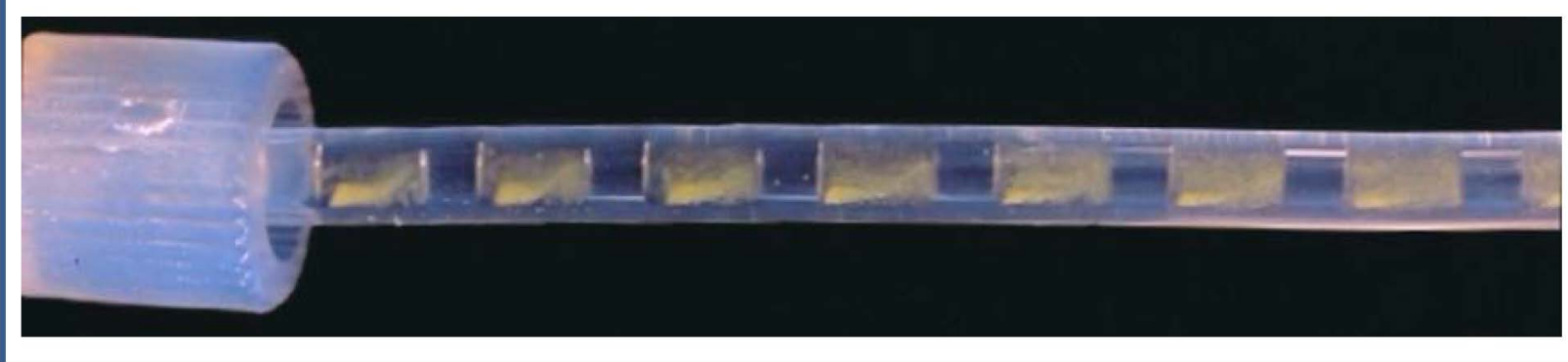

A flow system for processing solids enables the efficient and scalable utilization of Heterogeneous photocatalysts for selective fluorinations.

This article may be used for non-commercial purposes in accordance with Wiley Terms and Conditions for Self-Archiving. 


\title{
Continuous Heterogeneous Photocatalysis in Serial Micro-Batch
}

\section{Reactors}

\author{
Bartholomäus Pieber ${ }^{[a]}$, Menny Shalom ${ }^{[b] \dagger}$, Markus Antonietti ${ }^{[b]}$, Peter H. Seeberger ${ }^{\star[a]}$, Kerry Gilmore ${ }^{\star[a]}$
}

\begin{abstract}
Solid reagents, leaching catalysts, and heterogeneous photocatalysts are commonly employed in batch processes but are ill suited for continuous flow chemistry. Heterogeneous catalysts for thermal reactions are typically used in packed bed reactors that cannot be penetrated by light and thus are not suitable for photocatalytic reactions involving solids. We demonstrate that serial micro-batch reactors (SMBRs) allow for the continuous utilization of solid materials together with liquids and gases in flow. This technology was utilized to develop selective and efficient fluorination protocols using a modified graphitic carbon nitride heterogeneous catalyst instead of costly homogeneous metal polypyridyl complexes The merger of this inexpensive, recyclable catalyst and the SMBR approach gives access to sustainable and scalable photo catalysis.
\end{abstract}

Solid-liquid reactions are ubiquitous in chemical synthesis and are usually straightforward in batch, but they are less trivial in continuous flow devices. ${ }^{[1-4]}$ Packed bed reactors with heterogeneous catalysts embedded between filter units ${ }^{[1,5]}$ suffer from periodic deactivation or loss of catalyst due to leaching. Photochemistry on the other hand is perfectly suited for continuous processing as the large surface-to-volume ratio ensures an increased irradiation efficiency, resulting in intensified and scalable protocols. ${ }^{[1,6]}$ Visible-light photoredox catalysis $(\mathrm{PRC})^{[7-8]}$ employs costly ruthenium and iridium catalysts or organic molecules prone to degradation. ${ }^{[9-10]}$ Difficulties associated with separation and recycling of these catalysts render heterogeneous or easily recyclable catalysts such as modified transition metal catalysts ${ }^{[11-13]}$ and semiconductors ${ }^{[14-16]}$ highly desirable for PRC. Graphitic carbon nitrides $\left(\mathrm{g}-\mathrm{C}_{3} \mathrm{~N}_{4}\right)$, a class of metal-free polymers, are among the most promising materials for heterogeneous PRC. ${ }^{[17-18]}$ Combining the advantages of continuous photochemistry and g$\mathrm{C}_{3} \mathrm{~N}_{4}$ catalysis to provide an efficient and sustainable process is conceptually attractive but challenging practically. Packed bed reactors are not the method of choice as photons will be exclusively absorbed at the outer region while the inner particles are shielded (Fig. 1A). Small diameter packed bed reactors can overcome the irradiation problem ${ }^{[19]}$ but packing is tedious and channeling phenomena, as well as high pressure drops, are foreseeable drawbacks. ${ }^{[20]}$ Wall-coated reactors were previously utilized for continuous photocatalytic reactions, ${ }^{[6]}$ however,

[a] Dr. B. Pieber, Dr. K. Gilmore, Prof. Dr. P. H. Seeberger Department of Biomolecular Systems

Max Planck Institute of Colloids and Interfaces Am Mühlenberg 1, 14476 Potsdam, Germany E-mail: peter.seeberger@mpikg.mpg.de; kerry.gilmore@mpikg.mpg.de

[b] Dr. M. Shalom, Prof. Dr. M. Antonietti Department of Colloid Chemistry

Max Planck Institute of Colloids and Interfaces Am Mühlenberg 1, 14476 Potsdam, Germany

$\dagger \quad$ Current address: Chemistry Department, Ben Gurion University of the Negev, Beersheba 009728, Israe stable and homogeneously distributed wall coatings are difficult to prepare and different for each heterogeneous catalyst. Efficient irradiation is ensured by pumping a suspension through a coil reactor but the solid material settles, leading to a heterogeneous distribution, irreproducible results, or clogging (Fig. 1B).

Dosing a catalyst suspension into a segmented gasliquid stream should, based on suspension feeding into segmented flow regimes, ${ }^{[20-22]}$ provide a promising alternative for heterogeneous photocatalysis. Such triphasic systems resemble a series of small solid-liquid batch reactors (serial micro-batch reactors - SMBRs) that are separated by an inert gas spacer and "stirred" by toroidal currents (Fig. 1C)

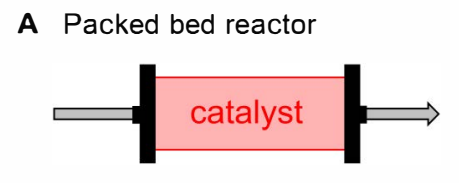

B Pumping a suspension

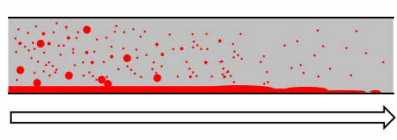

C Triphasic system (this work)

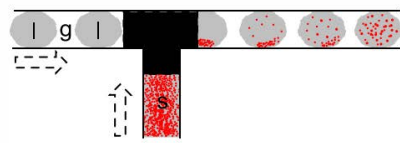

+ Solid separation included

- Light penetration

- Deactivation/leaching

- Channeling \& pressure

I..........................
- No solid separation

+ Light penetration

- Settling of solids/clogging

- Irreproducible

+ Light penetration

+ Internal mixing

+ All conditions adjustable
- No solid separation

Figure 1. Strategies for heterogeneous photocatalysis in flow. (A) Packed bed reactors are ideal for heterogeneous catalysis but suffer from insufficient light penetration. (B) Pumping a solid-liquid suspension is troublesome as the material settles and aggregates. (C) A triphasic gas-liquid-solid system combines the advantages of batch and flow processing for heterogeneous photocatalysis.

Earlier solid feeding systems suffer from complex setups $^{[20-21]}$ or costly equipment ${ }^{[22]}$ and are generally not suitable for continuous operation. ${ }^{[4]}$ To ensure straightforward access for the general community, SMBR generation was designed to be operationally simple and readily assembled from inexpensive, commercially available parts. The residence time and catalyst stoichiometry should be adjustable to enable detailed studies of all reaction parameters.

A flow setup for transformations on small scales meeting all requirements is a dosing system comprised of two parts. First, a stable gas-liquid segmented flow pattern is generated from the reaction solvent and nitrogen gas in a fluorinated ethylene propylene tube using a Y-shaped mixer (Fig. 2A). Subsequently, the catalyst suspension is added from below via a mixing Tee and a vertically mounted syringe pump that incorporates a magnetic stir bar. The resulting SMBRs can be fed into an irradiated coil reactor that is submerged in a thermostatic bath The residence time is adapted by changing the gas and/or liquid flow rate or the reactor volume while the catalyst stoichiometry 


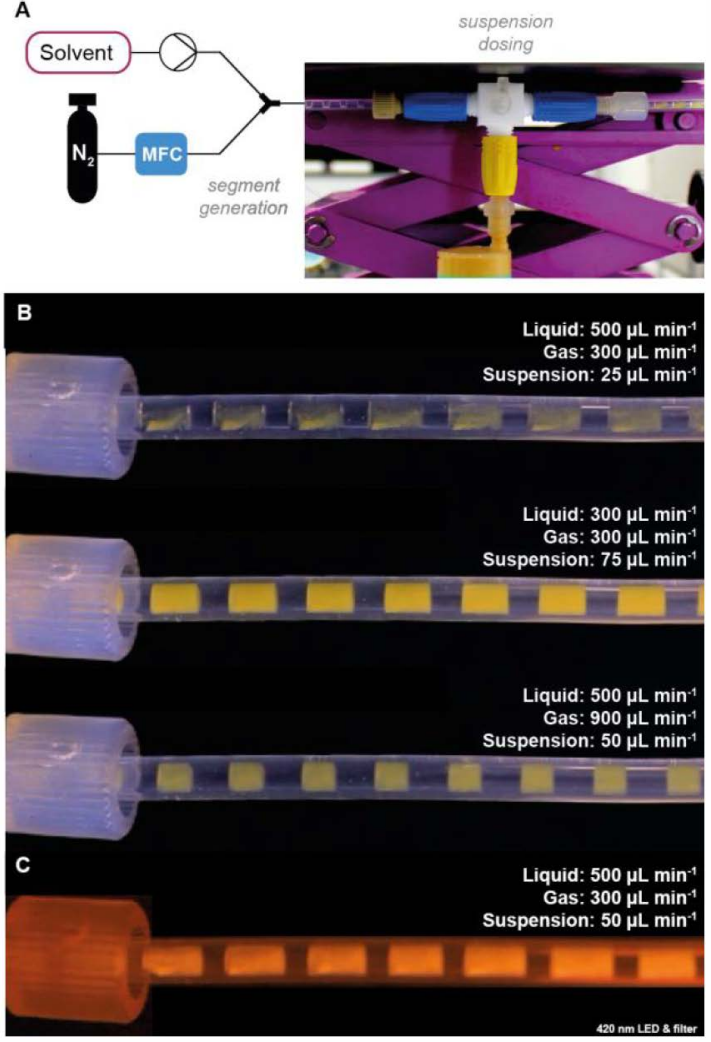

Figure 2. Continuous generation of serial micro-batch reactors (SMBRs). (A) Schematic representation of the solid dosing system. MFC = mass flow controller. (B) On the fly adjustment of reaction parameters. (C) Internal mixing results in a uniform, efficiently irradiated suspension (segments were irradiated with $420 \mathrm{~nm}$ LEDs and the picture was taken through a blue light filter). See Supplementary Information for experimental details.

can be varied by changing the rate of suspension dosing (Supporting Information). The system continuously produces identical SMBRs while all parameters can be changed during operation. The installation of a second catalyst addition unit enables a fully continuous operation by mimicking a dual syringe pump (Supporting Information).

A modified carbon nitride $\left(\mathrm{CMB}-\mathrm{C}_{3} \mathrm{~N}_{4}\right)$ that is highly efficient for the photocatalytic production of hydrogen from water served as heterogeneous photocatalyst for initial tests. ${ }^{[23]}$ This bench-stable semiconductor can be prepared in large quantities via a supramolecular approach from bulk chemicals and absorbs in the visible region. ${ }^{[23]} \mathrm{CMB}-\mathrm{C}_{3} \mathrm{~N}_{4}$ shows minimal settling in viscous solvents such as ethylene glycol and 1-butyl-3methylimidazolium tetrafluoroborate $\left(\left[\mathrm{Bmim}_{\mathrm{B}} \mathrm{BF}_{4}\right)\right.$ a basis for the reliable preparation of a stable suspension for the accurate dosing of solid materials. Active mixing of the suspended catalyst feed via mechanical stirring improves reproducible additions, as negligible fluctuations over the course of addition were observed. The internal mixing phenomena caused by interior vortices inherent to the SMBRs result in a homogeneous and uniformly irradiated suspension of the semiconductor (Supporting Information).

With the robust dosing system in place, the SMBR concept was illustrated for heterogeneous photocatalysis. The well documented decarboxylative fluorination of phenoxyacetic acids using Selectfluor under PRC conditions ${ }^{[24]}$ served as the model transformation, as the triphasic SMBR system is perfectly suited for reactions involving gaseous reagents and/or byproducts. Batch experiments revealed that $\mathrm{CMB}-\mathrm{C}_{3} \mathrm{~N}_{4}$ can substitute $\mathrm{Ru}(\mathrm{bpy})_{3}{ }^{2+}$ in the decarboxylative fluorination of phenoxyacetic acid (1) and can be reused several times without losing its catalytic activity (Supporting information). Stirring is of utmost importance for an efficient, reproducible batch process and the reaction rate drops on larger scales.

Under SMBR conditions, the drawbacks associated with stirring can be elegantly addressed while the high irradiation efficiency inherent of the small dimensions simultaneously enhances the catalytic process. Monofluorinated compound 2 is almost quantitatively formed within only 14 minutes (Fig. 3A). The generated $\mathrm{CO}_{2}$ can immediately diffuse out of the liquid phase, to form expanded gas slugs and increase the overall flow rate without disturbing the integrity of the solid-liquid SMBRs (Fig. 3B). Following the reaction, a three-step extraction protocol provides the desired organofluorine compound without chromatography and allows for both the catalyst and suspension solvent $\left(\left[\mathrm{Bmim}_{\mathrm{B}} \mathrm{BF}_{4}\right)\right.$ to be fully recovered (Fig. $\left.3 \mathrm{C}\right)$.
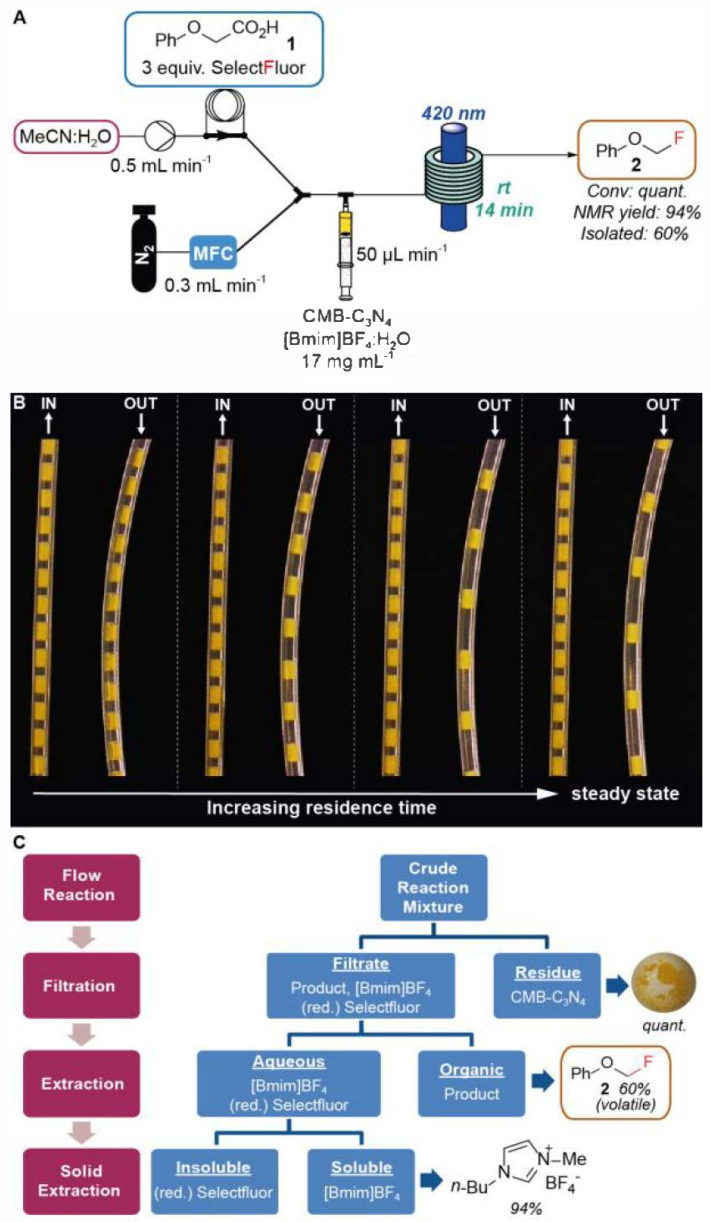

Figure 3. Heterogeneous photoredox catalysis with $\mathrm{CMB}-\mathrm{C}_{3} \mathrm{~N}_{4}$ in SMBRs. (A) Decarboxylative fluorination of phenoxyacetic acid. MFC $=$ mass flow controller. NMR yields were determined by ${ }^{19} \mathrm{~F}-\mathrm{NMR}$ using 2,2,2trifluoroethanol as internal standard. $(\mathrm{B}) \mathrm{CO}_{2}$ byproduct expands the gas slugs while keeping the solid-liquid SMBR intact. (C) Separation strategy for product isolation and recovery of the catalyst and $\left[\mathrm{Bmim}_{B} \mathrm{BF}_{4}\right.$. See Supporting Information for experimental details. 
The process can be easily scaled for the gram scale synthesis of 2 using a setup with two catalyst addition units (Supporting Information). Control experiments in the absence of either the semiconducting material or light did not result in an observable gas formation and only trace amounts of 2 were identified by NMR spectroscopy (Fig. 4A). Even when the reaction mixture was not degassed, the SMBR approach still produced 2 in excellent yield, whereas no reaction occurred in batch under similar conditions. Using compressed air instead of nitrogen for segment generation resulted in a $36 \%$ NMR yield and only pure oxygen completely inhibited the photocatalytic cycle. The "in situ degassing" of the SMBR system may occur via diffusion of dissolved oxygen into the gas segments. When $\mathrm{Ru}(\mathrm{bpy})_{3}{ }^{2+}$ was used instead of $\mathrm{CMB}-\mathrm{C}_{3} \mathrm{~N}_{4}$ employing a segmented flow pattern without prior degassing of the solution product was formed, but the homogeneous catalyst suffered from significantly lower conversion than the heterogeneous PRC. Moreover, reactor fouling with the ruthenium PRC resulted in a random flow profile over time (Supporting Information).
Homogeneous PRC fail for continuous chemistry on larger scale due to irreproducible reaction parameters and the high risk of clogging. The SMBR fluorodecarboxylation produced a range of phenoxyacetic acid derivatives (Fig. 4B). The hydrophobic nature of most products necessitated an acetone:water solvent mixture to avoid the generation of an "organic film" on the surface of the FEP coil (Supporting Information). Higher catalyst loading in combination with longer reaction times (up to $48 \mathrm{~min}$ ) ensured sufficient conversions and yields similar to those obtained by the original protocol, ${ }^{[24]}$ as well as the uncatalyzed version using UV light. ${ }^{[25]}$

The catalytic system was amenable to other fluorinations (Fig. 4C). The decarboxylative fluorination of 4 biphenylacetic acid (3) relied previously on a strongly oxidizing iridium(III) photocatalyst $\left(\operatorname{Ir}\left[\mathrm{dF}\left(\mathrm{CF}_{3}\right) \text { ppy }\right]_{2}(\mathrm{dtbbpy}) \mathrm{PF}_{6}\right)$ in presence of a base. ${ }^{[26]}$ Under SMBR conditions, 3 was converted to $4\left(73 \%\right.$ yield) within $40 \mathrm{~min}$ at $50{ }^{\circ} \mathrm{C}$ without additional reagents.

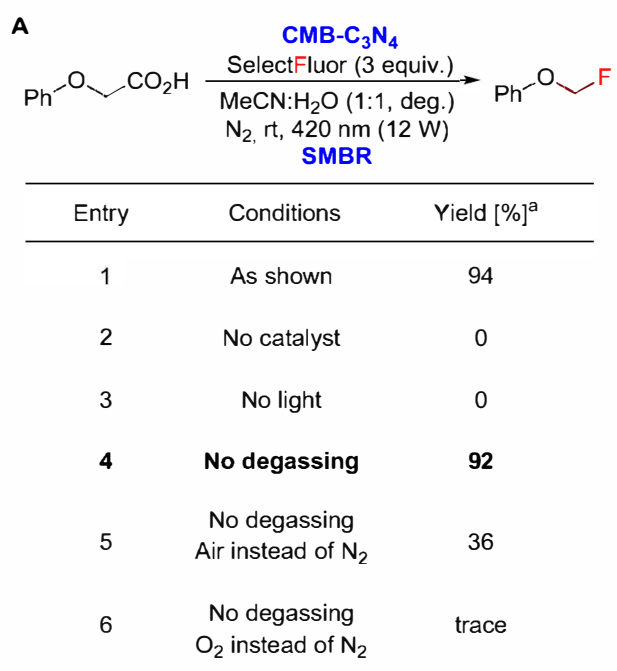

B

$$
\begin{aligned}
& \text { CMB-C } \mathrm{C}_{3}
\end{aligned}
$$

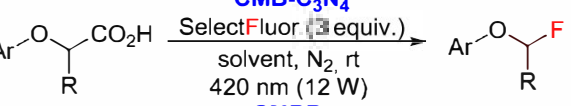
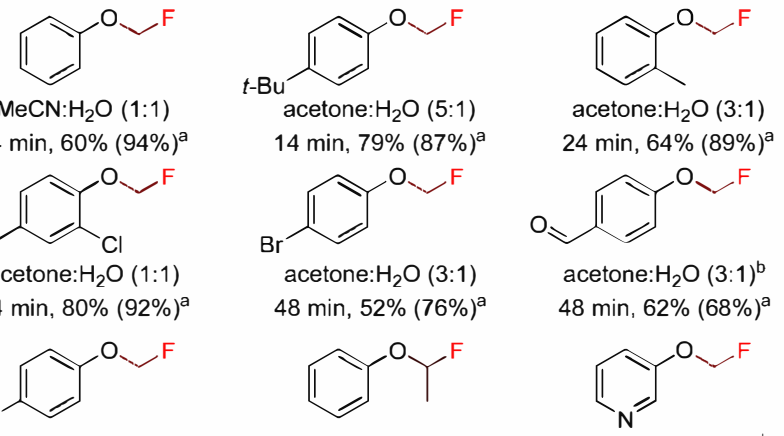

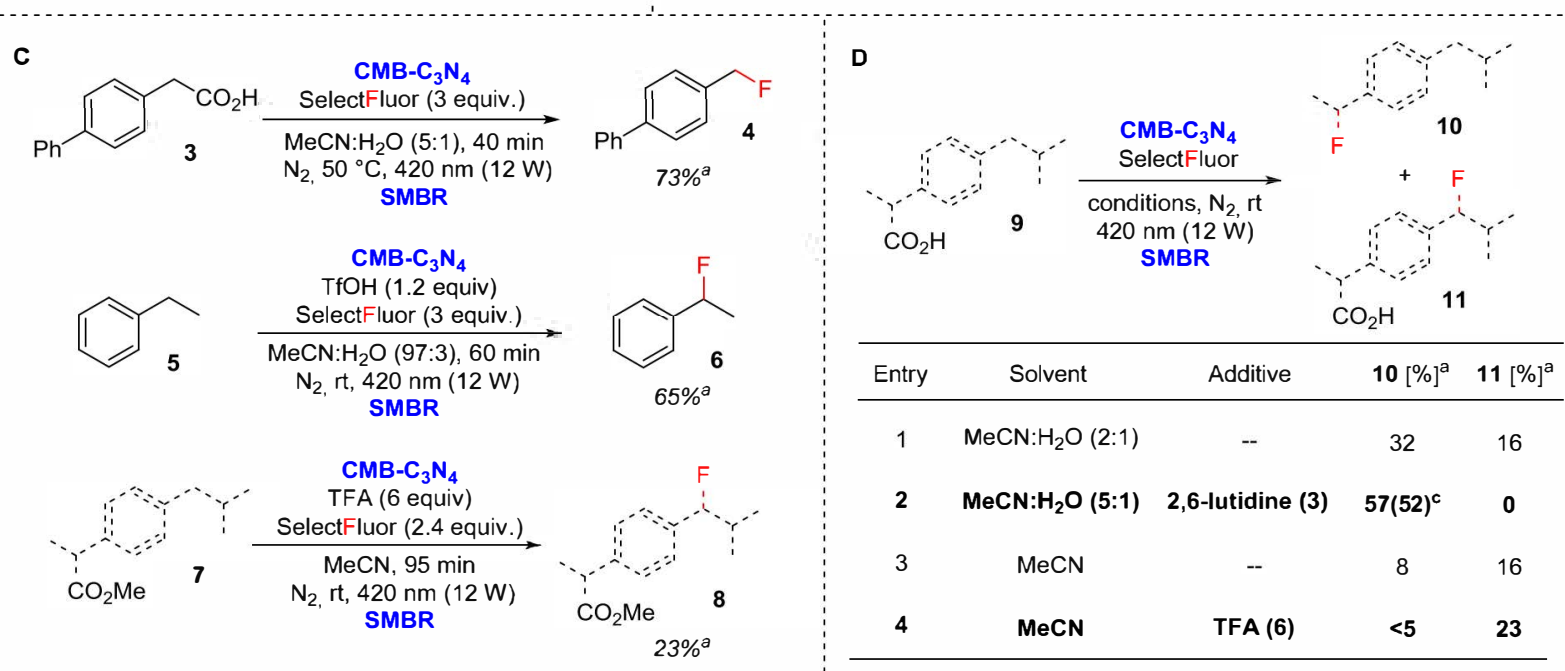

Figure 4. Application of SMBR to heterogeneous photoredox catalysis. (A) Control experiments indicate an "in situ degassing" effect of the triphasic reaction system. (B) Scope of the decarboxylative fluorination of phenoxyacetic acid derivatives. (C) Decarboxylative fluorination of 4-biphenylacetic acid and direct monofluorination of ethylbenzene and ibuprofen methyl ester. (D) Selectivity studies for the fluorination of ibuprofen. ${ }^{a}$ NMR yields are given in parenthesis. ${ }^{b} 2,6$-lutidine was added to dissolve the starting material. ' Isolated yield in parenthesis. See Supporting Information for experimental details. 
The direct benzylic monofluorination of ethylbenzene (5) was achieved using the heterogeneous photocatalyst and Selectfluor (5, Fig. 4D), a transformation usually carried out using photosensitizers such as 9-fluorenone. ${ }^{[27]}$ The $45 \%$ yield of the reaction without any additive was significantly increased by addition of a strong acid (65\%). The fluorination of ibuprofen methyl ester (7) was carried out under water-free conditions to avoid ester hydrolysis.

Selective late-stage fluorination of ibuprofen (9) at two different positions proved possible (Fig. 4E). The decarboxylative fluorination product 10 and benzylic $\mathrm{C}-\mathrm{H}$ fluorination product $\mathbf{1 1}$ were identified by NMR analysis. In presence of a base, only 10 was selectively obtained in moderate yield. Without water as cosolvent under acidic conditions, the decarboxylative fluorination pathway was suppressed, to produce mainly 9 (Supporting Information).

In summary, we have developed a flow chemistry system that combines the advantages batch processing offers for heterogeneous materials with the benefits of flow photochemistry. The simple and efficient continuous utilization of heterogeneous photocatalysts serves as example and can be extended to many solid catalysts and reagents where packed bed reactors are non-ideal. The system is well suited for reactions involving gases. Three different fluorination protocols utilizing a heterogeneous organic semiconductor - which holds numerous advantages over standard metal catalysts - were developed and the methodology proved efficient, flexible, and robust on various scales and allowed for the site-selective, latestage fluorination of ibuprofen. The SMBR concept, in combination with graphitic carbon nitride photocatalysis, will contribute to the development of more sustainable and industrially relevant photoredox processes in the future.

\section{Acknowledgements}

We gratefully acknowledge the Max-Planck Society for generous financial support. We gratefully acknowledge our colleagues Günter Haseloff, Jesús Barrio Hermida, Ines Cazin, Xuan Pham, and Dr. Daniel Varón Silva for technical, synthetic, and analytical support and Dr. Matthew B. Plutschack, Dr. Jiawei Rong, and Dr Martina Delbianco for fruitful discussions.

Keywords: flow chemistry - heterogeneous photocatalysis • carbon nitride $\cdot$ fluorination

[1] M. B. Plutschack, B. Pieber, K. Gilmore, P. H. Seeberger, Chem. Rev. 2017, 117, 11796-11893

[2] K. S. Elvira, X. C. i Solvas, R. C. R. Wootton, A. J. deMello, Nat. Chem. 2013, 5, 905-915.

[3] R. L. Hartman, Org. Process Res. Dev. 2012, 16, 870-887

[4] K. Wu, S. Kuhn, Chim. Oggi 2014, 32, 62-66.

[5] T. Tsubogo, H. Oyamada, S. Kobayashi, Nature 2015, 520, 329-332.

[6] D. Cambié, C. Bottecchia, N. J. W. Straathof, V. Hessel, T. Noël, Chem Rev. 2016, 116, 10276-10341.

[7] D. M. Schultz, T. P. Yoon, Science 2014, 343, 1239176.

[8] M. H. Shaw, J. Twilton, D. W. C. MacMillan, J. Org. Chem. 2016, 81, 6898-6926.
[9] A. Joshi-Pangu, F. Lévesque, H. G. Roth, S. F. Oliver, L.-C. Campeau, D. Nicewicz, D. A. DiRocco, J. Org. Chem. 2016, 81, 7244-7249.

[10] N. A. Romero, K. A. Margrey, D. A. Nicewicz, Science 2015, 349, 1326 1330.

[11] J. Guerra, D. Cantillo, C. O. Kappe, Catal. Sci. Tech. 2016, 6, 4695 4699.

[12] N. Priyadarshani, Y. Liang, J. Suriboot, H. S. Bazzi, D. E. Bergbreiter ACS Macro Lett. 2013, 2, 571-574

[13] D. Rackl, P. Kreitmeier, O. Reiser, Green Chem. 2016, 18, 214-219.

[14] D. Friedmann, A. Hakki, H. Kim, W. Choi, D. Bahnemann, Green Chem. 2016, 18, 5391-5411.

[15] X. Lang, X. Chen, J. Zhao, Chem. Soc. Rev. 2014, 43, 473-486.

[16] J. Chen, J. Cen, X. Xu, X. Li, Catal. Sci. Tech. 2016, 6, 349-362.

[17] X. Wang, S. Blechert, M. Antonietti, ACS Catal. 2012, 2, 1596-1606.

[18] J. Liu, Y. Liu, N. Liu, Y. Han, X. Zhang, H. Huang, Y- Lifshitz, S.-T. Lee, J. Zhong, Z. Khan, Science 2015, 347, 970-974

[19] M. Woźnica, N. Chaoui, S. Taabache, S. Blechert, Chem. Eur. J. 2014, 20, 14624-14628

[20] A. Ufer, D. Sudhoff, A. Mescher, D. W. Agar, Chem. Eng. J. 2011, 167, 468-474.

[21] A.-K. Liedtke, F. Bornette, R. Philippe, C. de Bellefon, Chem. Eng. J. 2013, 227, 174-181.

[22] K. Olivion, F. Sarrazin, Chem. Eng. J. 2013, 227, 97-102.

[23] M. Shalom, M. Guttentag, C. Fettkenhauer, S. Inal, D. Neher, A. Llobet M. Antonietti, Chem. Mater. 2014, 26, 5812-5818.

[24] M. Rueda-Becerril, O. Mahé, M. Drouin, M. B. Majewski, J. G. West, M O. Wolf, G. M. Sammis, J.-F. Paquin, J. Am. Chem. Soc. 2014, 136, 2637-2641.

[25] J. C. T. Leung, C. Chatalova-Sazepin, J. G. West, M. Rueda-Becerril J.-F. Paquin, G. M. Sammis, Angew. Chem. Int. Ed. 2012, 51, 1080410807.

[26] S. Ventre, F. R. Petronijevic, D. W. C. MacMillan, J. Am. Chem. Soc 2015, 137, 5654-5657

[27] J.-B Xia, C. Zhu, C. Chen, J. Am. Chem. Soc. 2013, 135, 17494-17500. 